\title{
Design of Stretchable Holey Gold Biosensing Electrode for Real-Time \\ Cell Monitoring
}

Yunzhi Ling ${ }^{1 \dagger}$, Quanxia Lyu ${ }^{1,2 \dagger}$, Qingfeng Zhai ${ }^{1,2}$, Bowen $Z u^{1}$, Shu Gong ${ }^{1}$, Tian Zhang ${ }^{1}$, Jennifer Dyson,

2,3 Wenlong Cheng ${ }^{1,2 *}$

${ }^{1}$ Department of Chemical Engineering, Monash University, Clayton, Victoria 3800, Australia;

${ }^{2}$ New Horizon Research Centre, Monash University, Clayton, Victoria 3800, Australia;

${ }^{3}$ Department of Biochemistry \& Molecular Biology, Biomedicine Discovery Institute, Clayton, Victoria 3800, Australia;

*Correspondence author. Email: W. L. Cheng (wenlong.cheng@monash.edu)

$\dagger$ Y. Ling and Q. Lyu contributed equally to this article. 
(a)

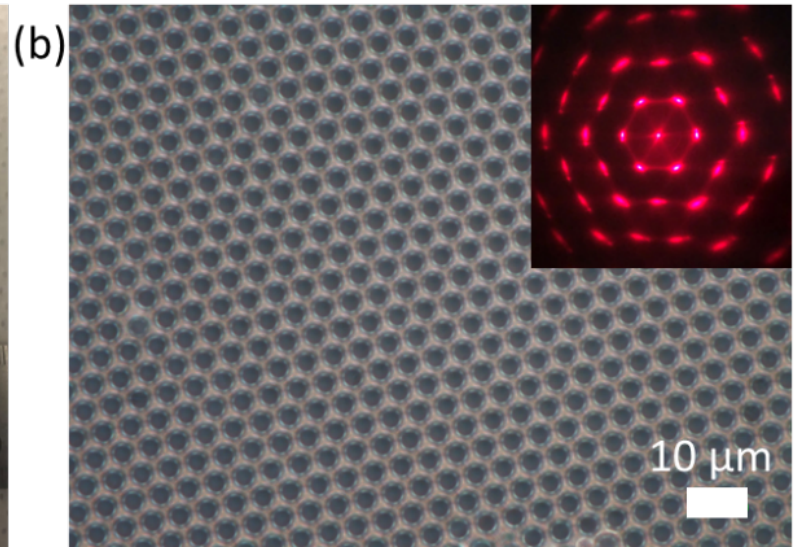

Figure S1 (a) Photograph of monolayer PS microspheres on PDMS surface, sample size: $6 \mathrm{~cm}$ $\times 5 \mathrm{~cm}$. (b) Microscopic image of PS microspheres array on PDMS surface, inset: diffraction pattern obtained by projecting a commercial laser pointer through the monolayer. 

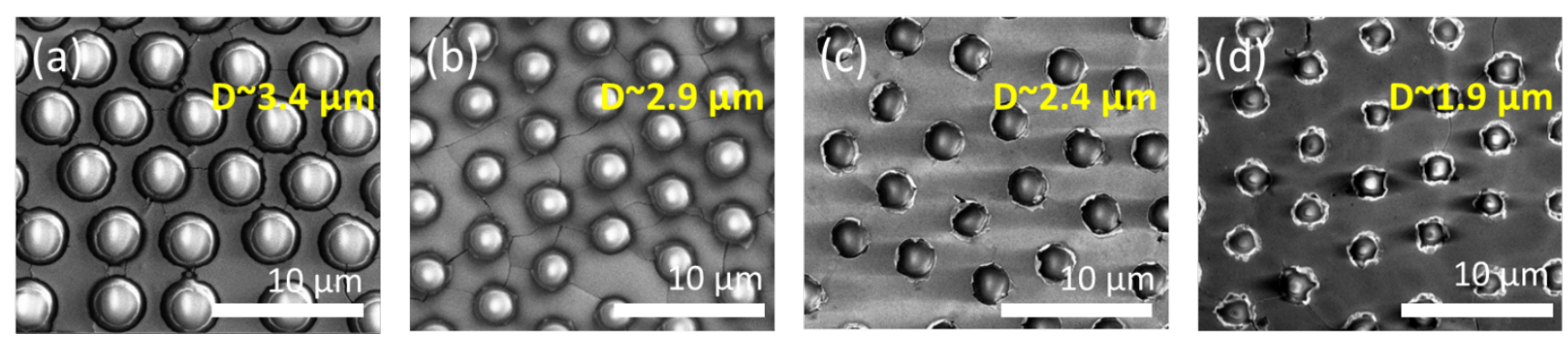

Figure S2 SEM images of stretchable pinhole gold electrode with different PS microsphere etching times (a) $5 \mathrm{~min}$; (b)10 min; (c)15 min and (d) $20 \mathrm{~min}$. The corresponding average diameters of pinhole are calculated at $3.4 \mu \mathrm{m}, 2.9 \mu \mathrm{m}, 2.4 \mu \mathrm{m}$ and $1.9 \mu \mathrm{m}$ respectively. 


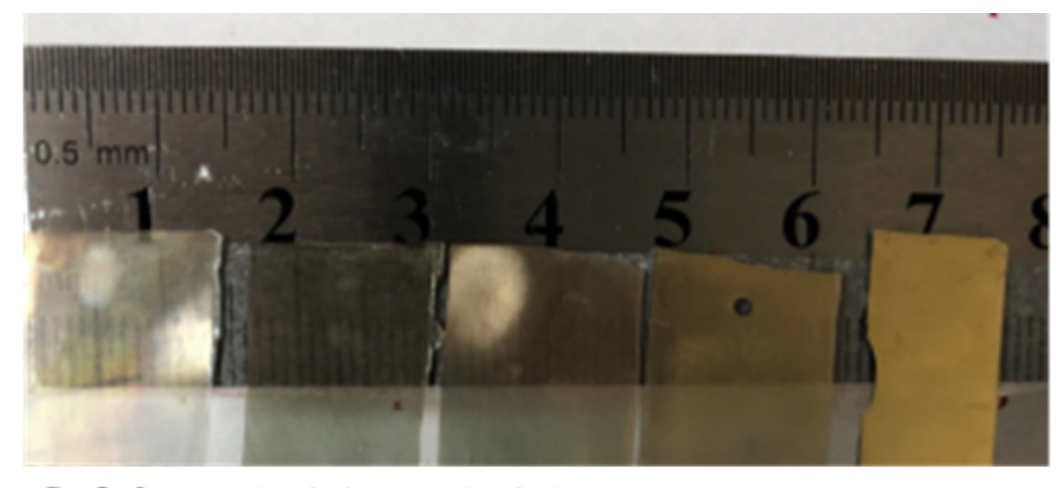

D 3.4 $\mu \mathrm{m} \quad$ D 2.9 $\mu \mathrm{m} \quad$ D 2.4 $\mu \mathrm{m} \quad$ D 1.9 $\mu \mathrm{m} \quad$ No pinhole

Figure S3 Optical images of stretchable pinhole gold electrode with different pinhole diameters. 


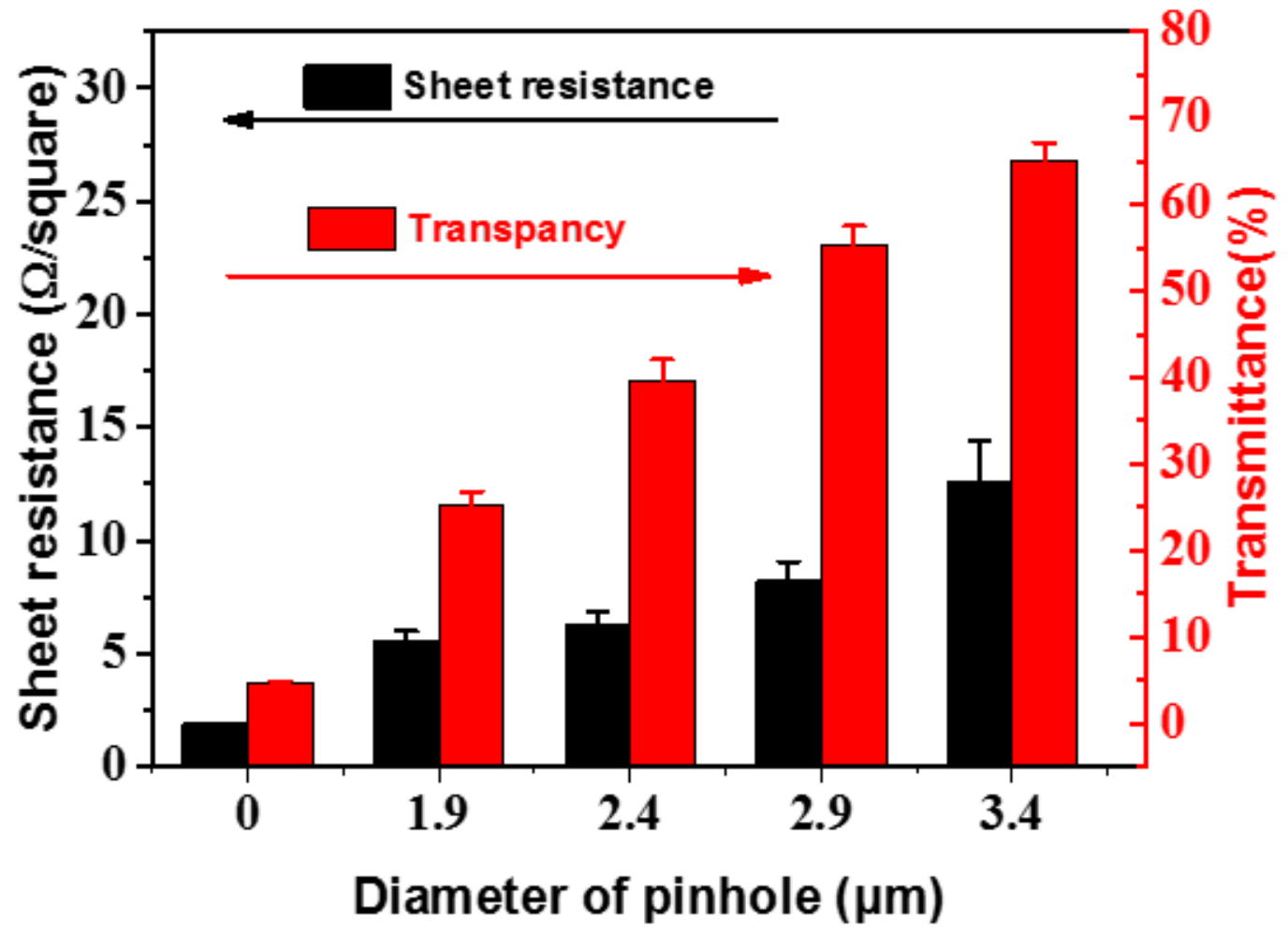

Figure S4 Comparisons between sheet resistance and transmittance as a function of pinhole diameter $(0 \mu \mathrm{m}, 1.9 \mu \mathrm{m}, 2.4 \mu \mathrm{m}, 2.9 \mu \mathrm{m}$ and $3.4 \mu \mathrm{m})$. 

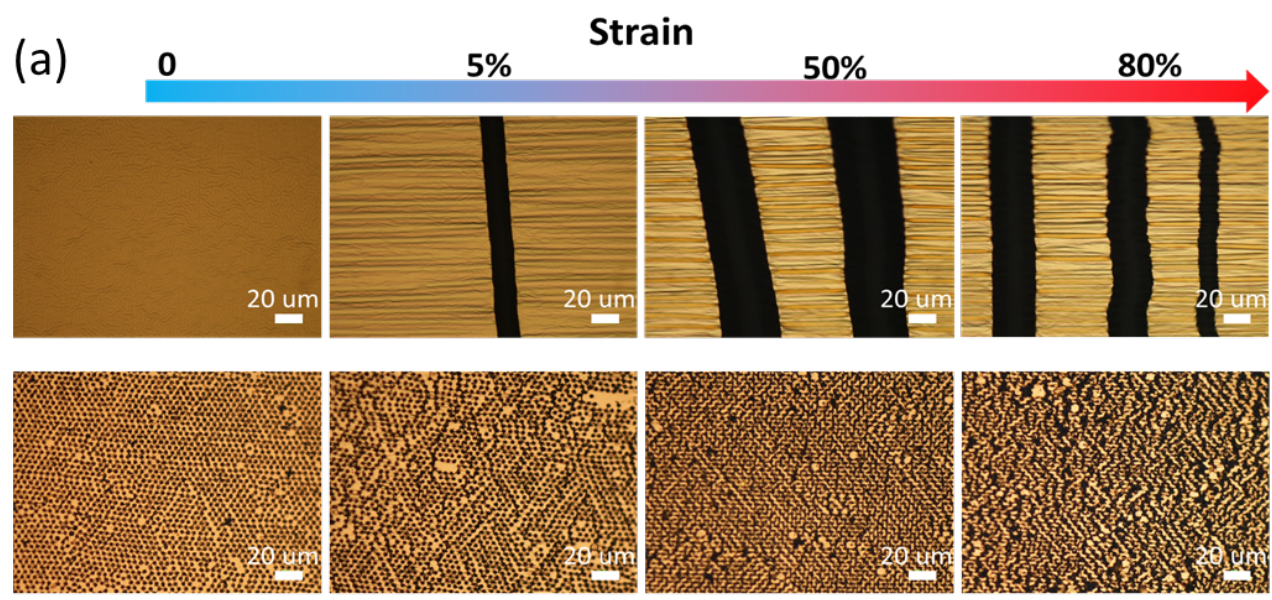

\section{Crack density}

(b)

(c)
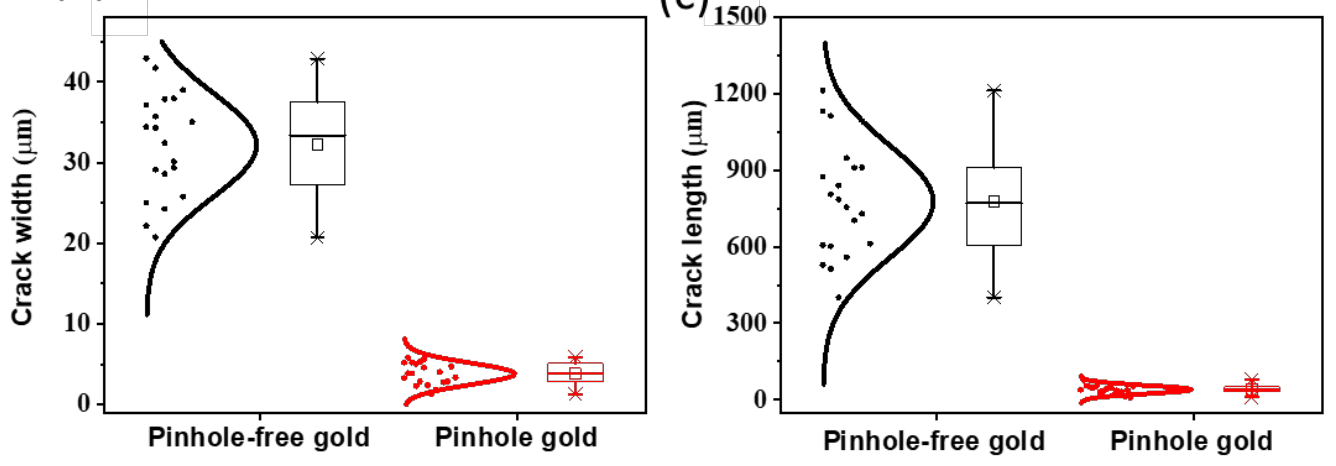

Figure S5 Crack comparison between pinhole-free gold electrode and pinhole gold electrode.

(a) Optical images of the pinhole-free gold electrode (top) and pinhole gold electrode (bottom) under different levels of stains. The statistical data of the length (b) and width (c) of the cracks under $50 \%$ strain. 


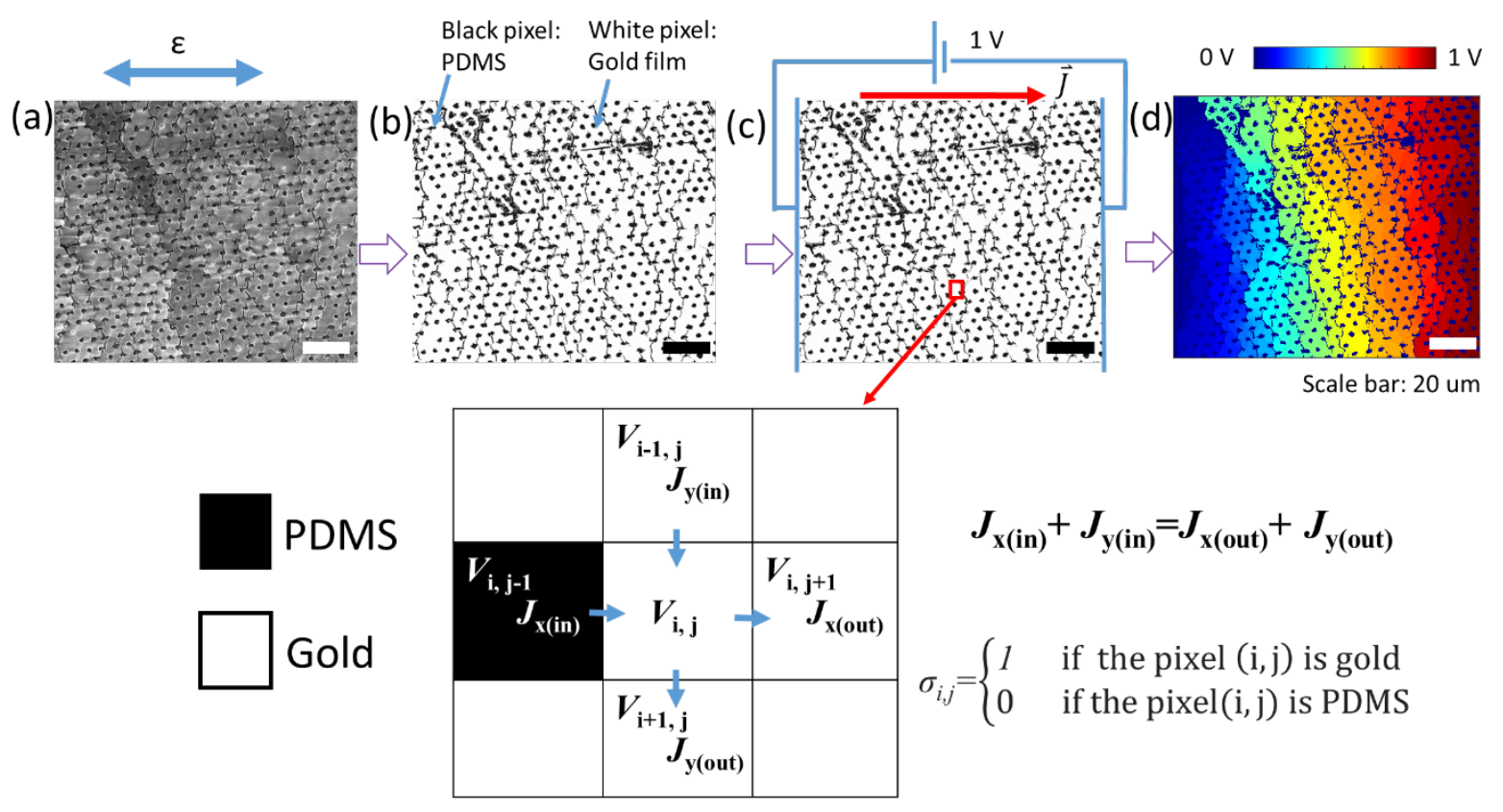

Figure S6 Simulation of voltage drop based on SEM images. (a) The original SEM image of pinhole gold film with cracks under strain. (b) Converted binary SEM, the white pixels indicate gold and the black pixels represent crack. (c) $1 \mathrm{~V}$ is applied at the left and right ends of image. Enlarged view: the current flow of pixel $V_{i, j}$ with the neighboring pixels. (d) The voltage distribution map of the original SEM. 


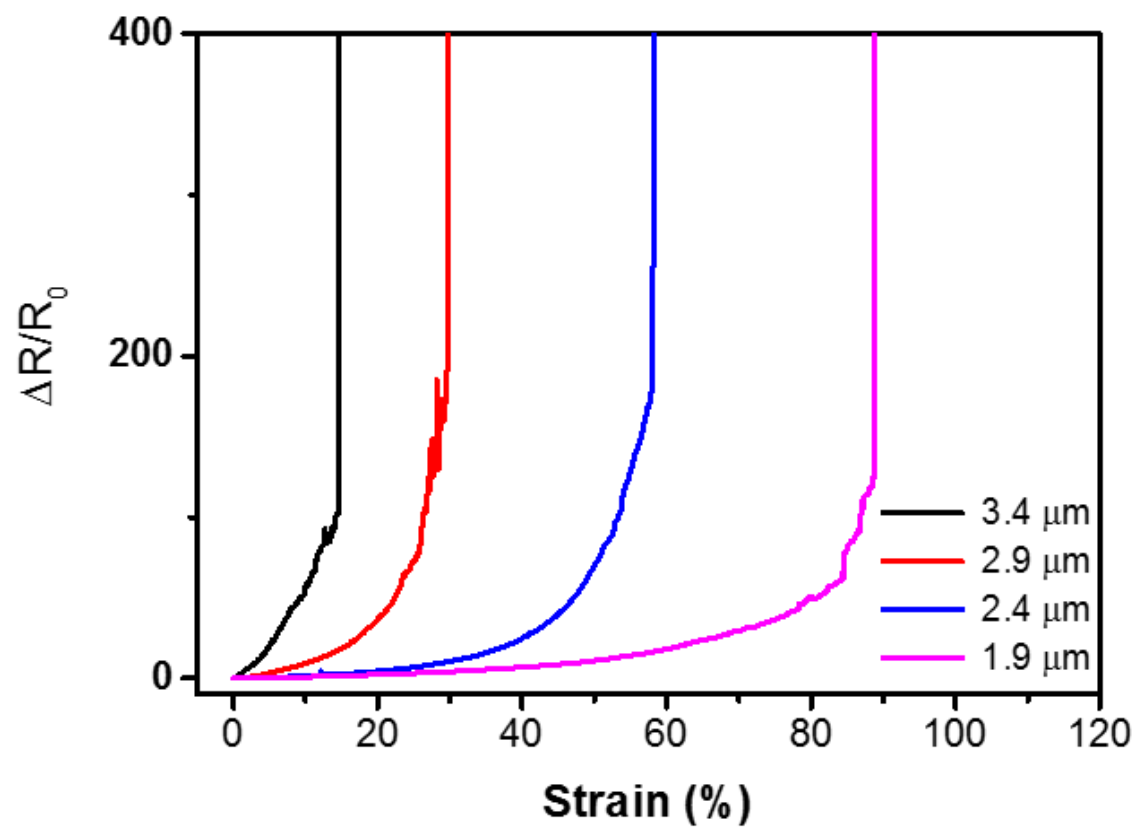

Figure S7 Stretch limitation of the stretchable pinhole gold electrode with different pinhole diameters. 

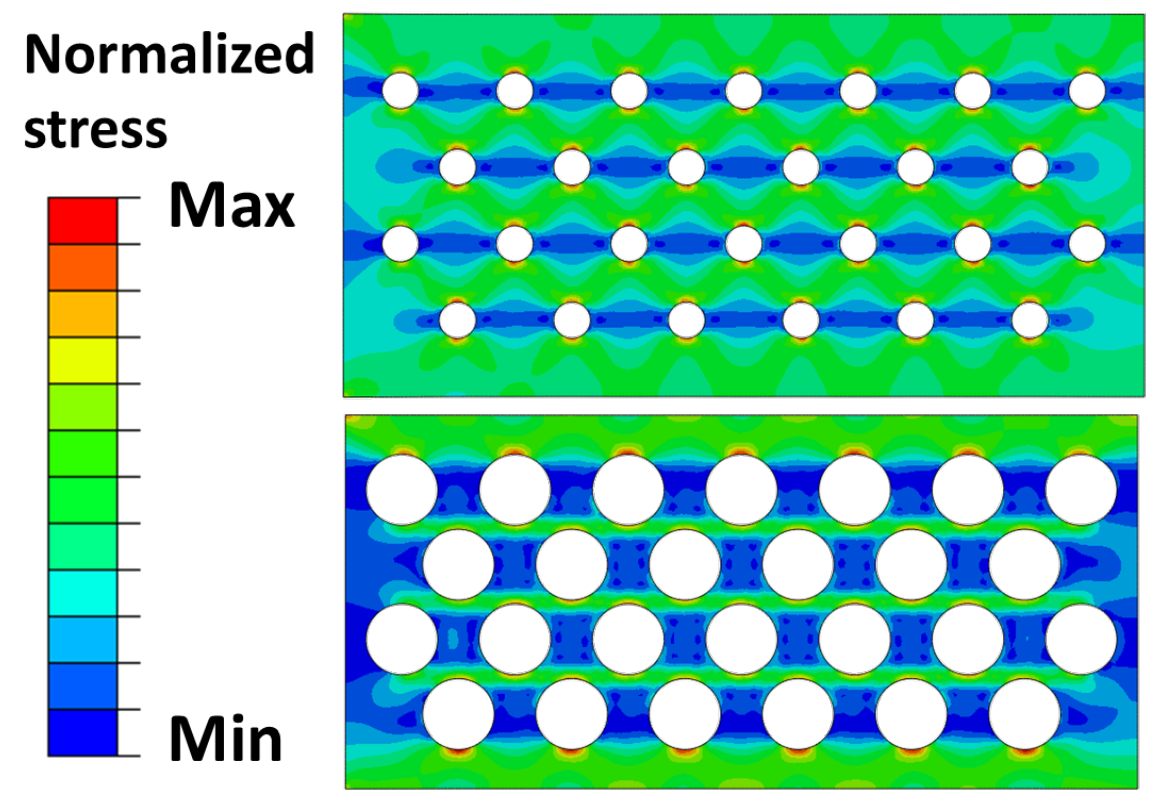

Figure S8 FEA simulation of meshed films with hole diameters of $1.9 \mu \mathrm{m}$ (top) and $3.9 \mu \mathrm{m}$ (bottom) upon 10\% strain applied. 


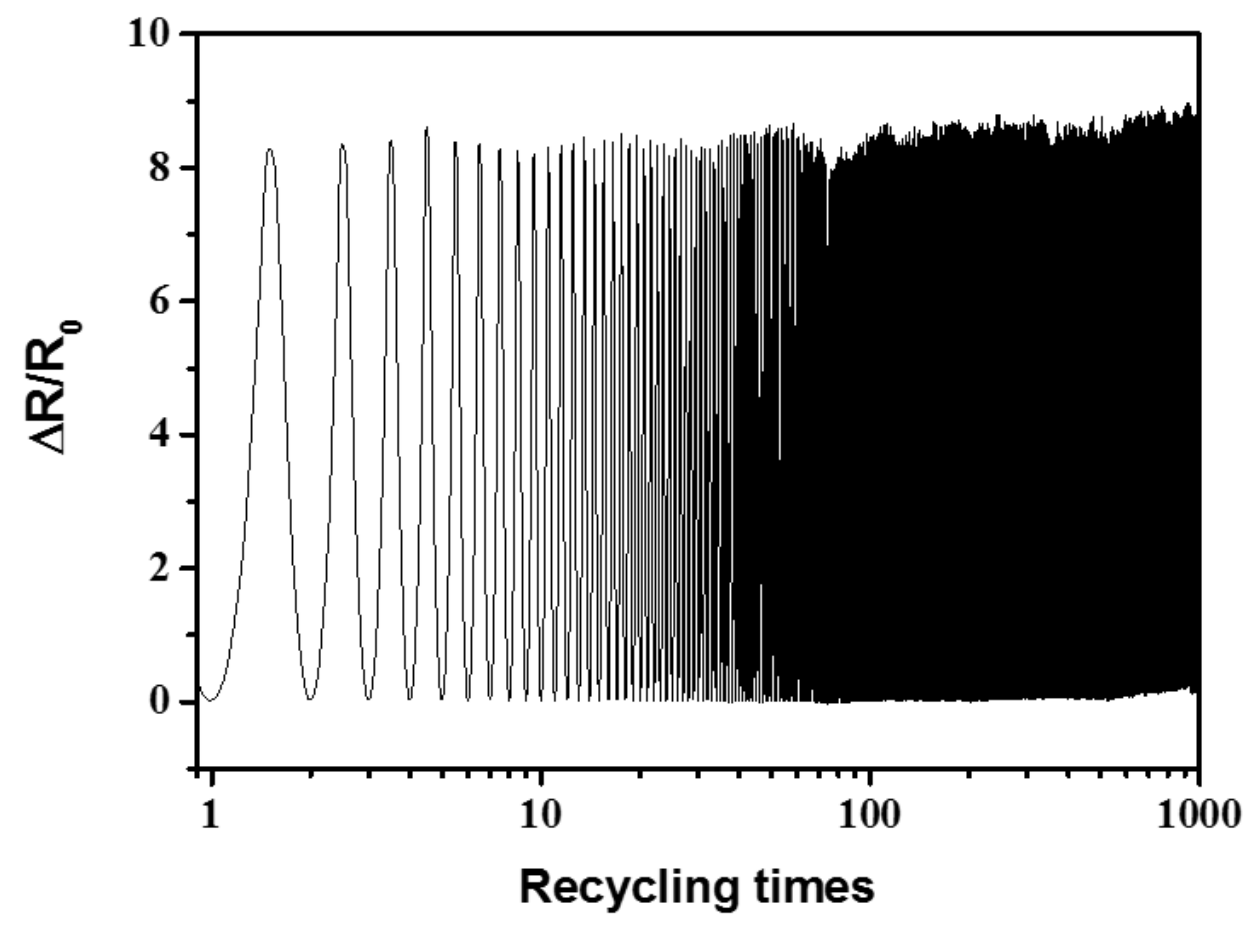

Figure S9 Durability test for the stretchable pinhole gold electrode under 50\% strain for 1000 cycles. 
(a)

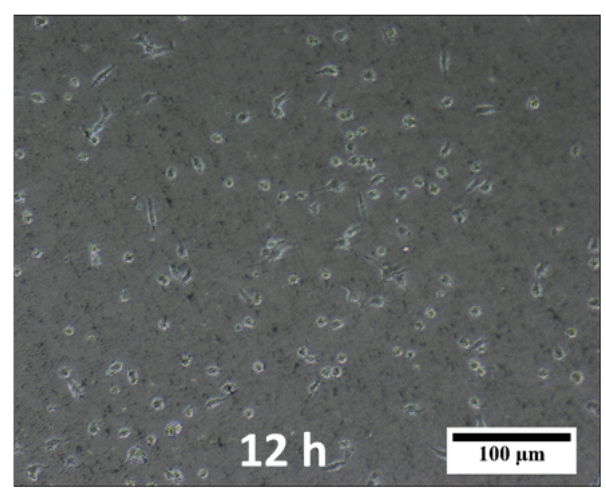

(c)

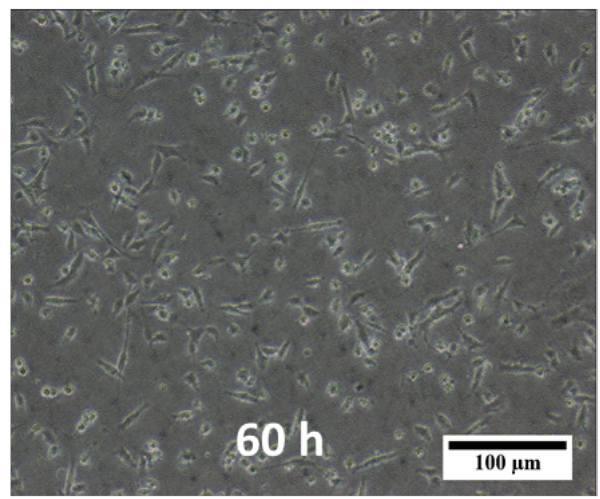

(b)

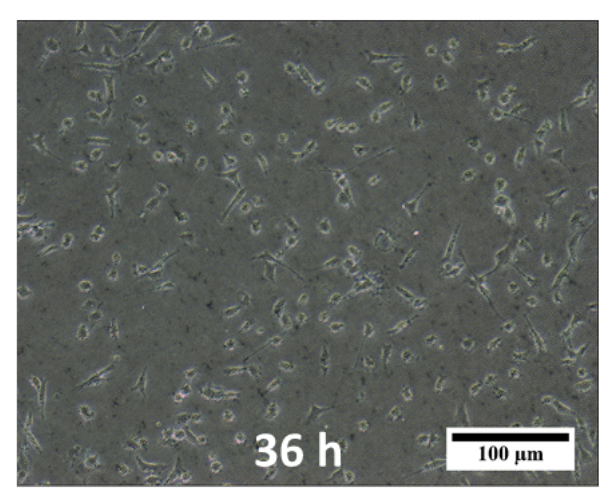

(d)

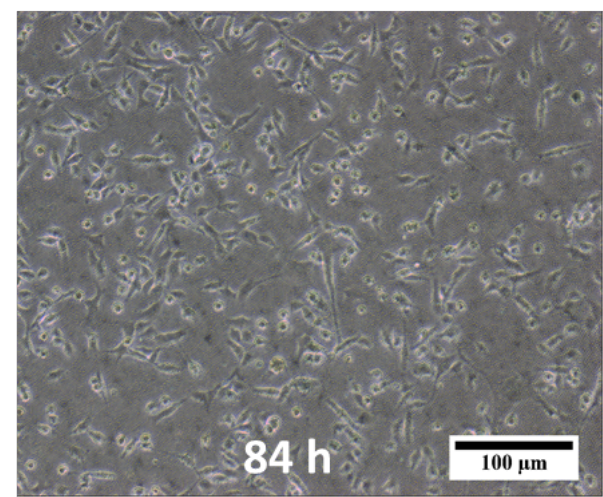

Figure S10 Phase-contrast images of MDA-MB-231 cells on the stretchable pinhole gold electrode at different plating times. Cells cultured on the pinhole gold electrode at a seeding density of $1 \times 10^{5}$ cells $/ \mathrm{cm}^{2}$ for (a) 12 hours, (b) 36 hours, (c) 60 hours and (d) 84 hours. 

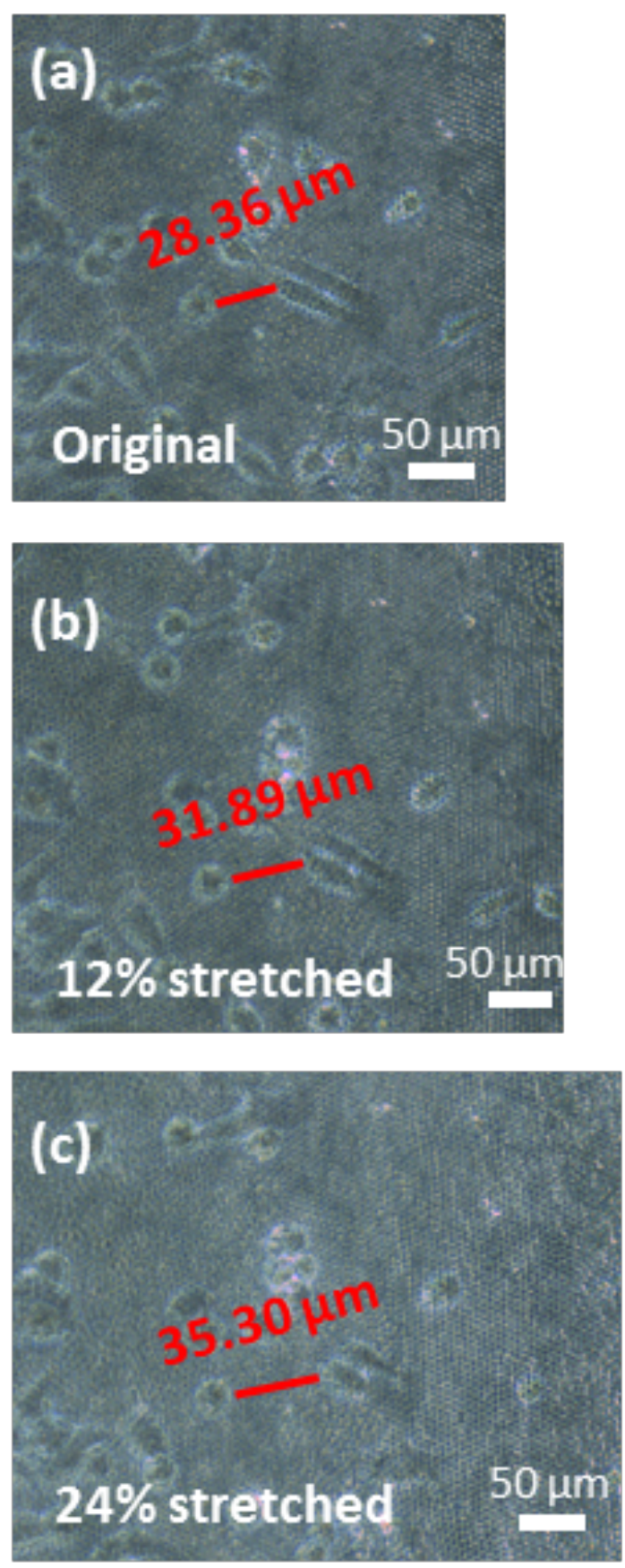

Figure S11 Phase-contrast images of cells on the stretchable pinhole gold electrode at different states. (a) Cells at original state, (b) $12.4 \%$ stretched state and (c) $24.4 \%$ stretched state. The distances between two adjacent cells are $28.36 \mathrm{~cm}, 31.89 \mathrm{~cm}$ and $35.30 \mathrm{~cm}$ in the three states respectively. 
Simulation of voltage drop based on SEM images: The mechanism of this simulation was explained by reference, where the authors assumed that each grid of SEM image followed the Ohm's law. ${ }^{1-3}$ The first step is to binarize gray SEM image to black-and-white color using Photoshop software (Figure S5a). The white pixels indicate gold and the black pixels represent crack, respectively. (Figure S5b) The conductivity of white pixel is set as 1 while the conductivity for black pixel is 0 . Later, we apply $1 \mathrm{~V}$ potential difference on two sides of binarized SEM. (Figure S5c) The current continuity equation is then applied to the image with each pixel being considered as a grid point. Ohm's law for the currents in each pixel is described as

$$
\mathrm{J}_{\mathrm{x}(\text { in })}+\mathrm{J}_{\mathrm{y}(\text { in })}=\mathrm{J}_{\mathrm{x}(\text { out })}+\mathrm{J}_{\mathrm{y}(\text { out })}
$$

Where $\mathrm{J}$ is the current intensity. To calculate the pixel potentials $\left(\mathrm{V}_{\mathrm{i}, \mathrm{j}}\right)$, the equation for all pixels are put in matrix format

$$
\mathrm{Ax}=\mathrm{b}
$$

Where $\mathrm{A}$ is the conductivity matrix of pixels, $\mathrm{x}$ is the column matrix of all the pixels and $\mathrm{b}$ is the boundary condition information. After processing matrix in Matlab, voltage distribution (x) can be obtained and shown in Figure S5d.

Supporting video

Dynamic simulation for hole-meshed and hole-free films during 20\% stretching process. 


\section{Reference}

1. W. Z. Cao, P. Gorrn and S. Wagner, Modeling the electrical resistance of gold film conductors on uniaxially stretched elastomeric substrates, Appl. Phys. Lett., 2011, 98, 212112.

2. V. Venugopalan, R. Lamboll, D. Joshi and K. S. Narayan, Facile Fabrication of UltraStretchable Metallic Nanocluster Films for Wearable Electronics, ACS Appl. Mater. Inter., 2017, 9, 28010-28018.

3. Y. Jiang, Z. Y. Liu, N. Matsuhisa, D. P. Qi, W. R. Leow, H. Yang, J. C. Yu, G. Chen, Y. Q. Liu, C. J. Wan, Z. J. Liu and X. D. Chen, Auxetic mechanical metamaterials to enhance sensitivity of stretchable strain sensors, Adv. Mater., 2018, 30, 1706589. 